\title{
Reactive Transport with Fluid-Solid Interactions in Dual-Porosity Media
}

\author{
Alon Nissan, Uria Alcolombri, ${ }^{*}$ Frédéric de Schaetzen, Brian Berkowitz, and Joaquin Jimenez-Martinez*
}

Cite This: ACS EST Water 2021, 1, 259-268

Read Online

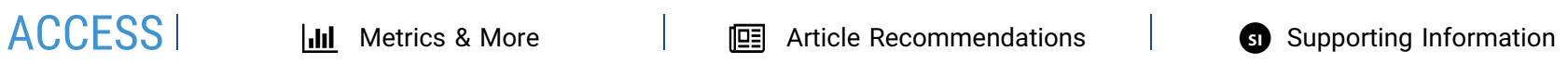

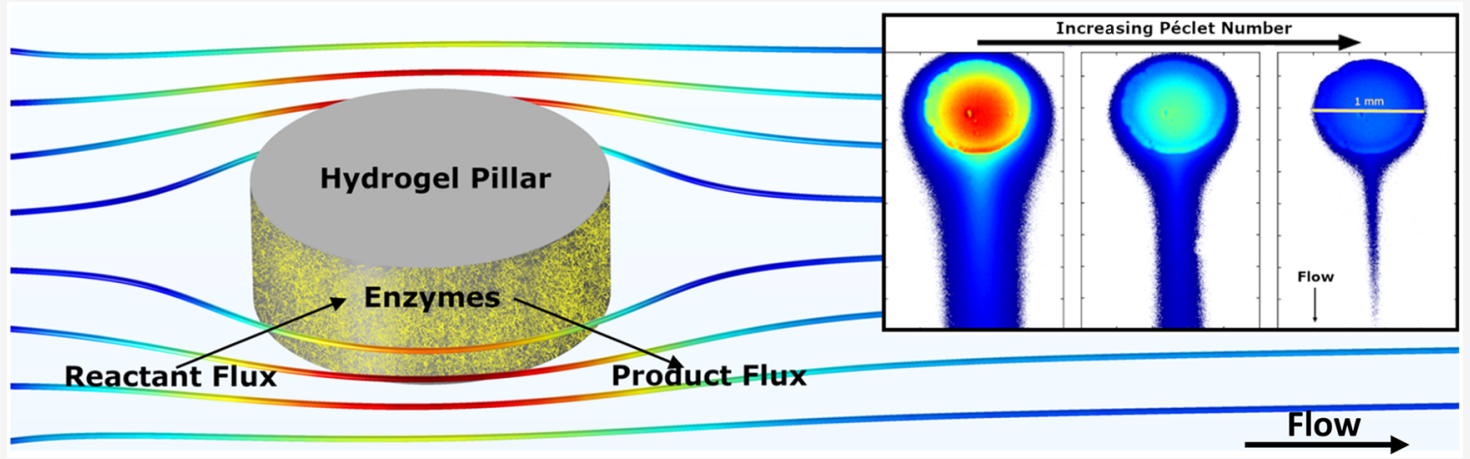

ABSTRACT: We study pore-scale dynamics of reactive transport in heterogeneous, dual-porosity media, wherein a reactant in the invading fluid interacts chemically with the surface of the permeable grains, leading to the irreversible reaction $A_{a q}+B_{s} \rightarrow C_{a q} . A$ microfluidic porous medium was synthesized, consisting of a single layer of hydrogel pillars (grains), chemically modified to contain immobilized enzymes on the grain surfaces. Fluorescence microscopy was used to monitor the spatiotemporal evolution of the reaction product $\mathrm{C}_{\mathrm{aq}}$ at different flow rates (Péclet values) and to characterize the impact on its transport. The experimental setup enables delineation of three key features of the temporal evolution of the reaction product within the domain: (i) the characteristic time until the rate of $\mathrm{C}_{\mathrm{aq}}$ production reaches steady state, (ii) the magnitude of the reaction rate at steady state, and (iii) the rate at which $\mathrm{C}_{\mathrm{aq}}$ is flushed from the system. These features, individually, are found to be sensitive to the value of the Péclet number, because of the relative impact of diffusion (vs advection) on the production and spatiotemporal evolution of $\mathrm{C}_{\mathrm{aq}}$ within the system. As the Péclet number increases, the production of $\mathrm{C}_{\mathrm{aq}}$ is reduced and the transport becomes more localized within the vicinity of the grains. The dual-porosity feature causes the residence time of the transported species to increase, by forming stagnant zones and diffusive-dominant regions within the flow field, thus enhancing the reaction potential of the system. Using complementary numerical simulations, we explore these effects for a wider range of Péclet and Damköhler numbers and propose nonlinear scaling laws for the key features of the temporal evolution of $\mathrm{C}_{\mathrm{aq}}$.

KEYWORDS: fluid flow dynamics, surface reaction, microfluidics, hydrogel, enzymes, scaling laws

\section{INTRODUCTION}

Transport and reactions of chemical species in porous materials occur in diverse environmental and industrial processes, such as those controlling nutrient spatial distribution and soil quality, erosion of rocks, decontamination of groundwater, subsurface $\mathrm{CO}_{2}$ sequestration, or waste and drinking water treatment. While in natural systems understanding how chemical species spread, mix, and interact with the host environment is of paramount concern for sustainable management and future predictions, in industrial systems it is of vital importance for improving their efficiency. These key factors-spreading, mixing, and chemical interactions-are controlled by the physical mechanisms of advection, diffusion, and reaction. ${ }^{1}$ In a different context, the governing mechanism(s) that dominates might vary and thus lead to different patterns of reaction and transport regimes. As an example, considering the abundance of microbial communities in natural porous media, ${ }^{2}$ soils for which advection is a dominant component may host communities that are spatially localized in the vicinity of preferential flow paths, as these regions have a higher nutrient transport efficiency. ${ }^{3}$ In contrast, in diffusion-dominated systems, the distribution of microbial life is likely to be more spatially uniform, such as in deep sediments. ${ }^{4}$

Received: June 24, 2020

Revised: November 25, 2020

Accepted: November 30, 2020

Published: December 9, 2020

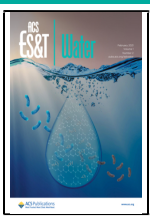



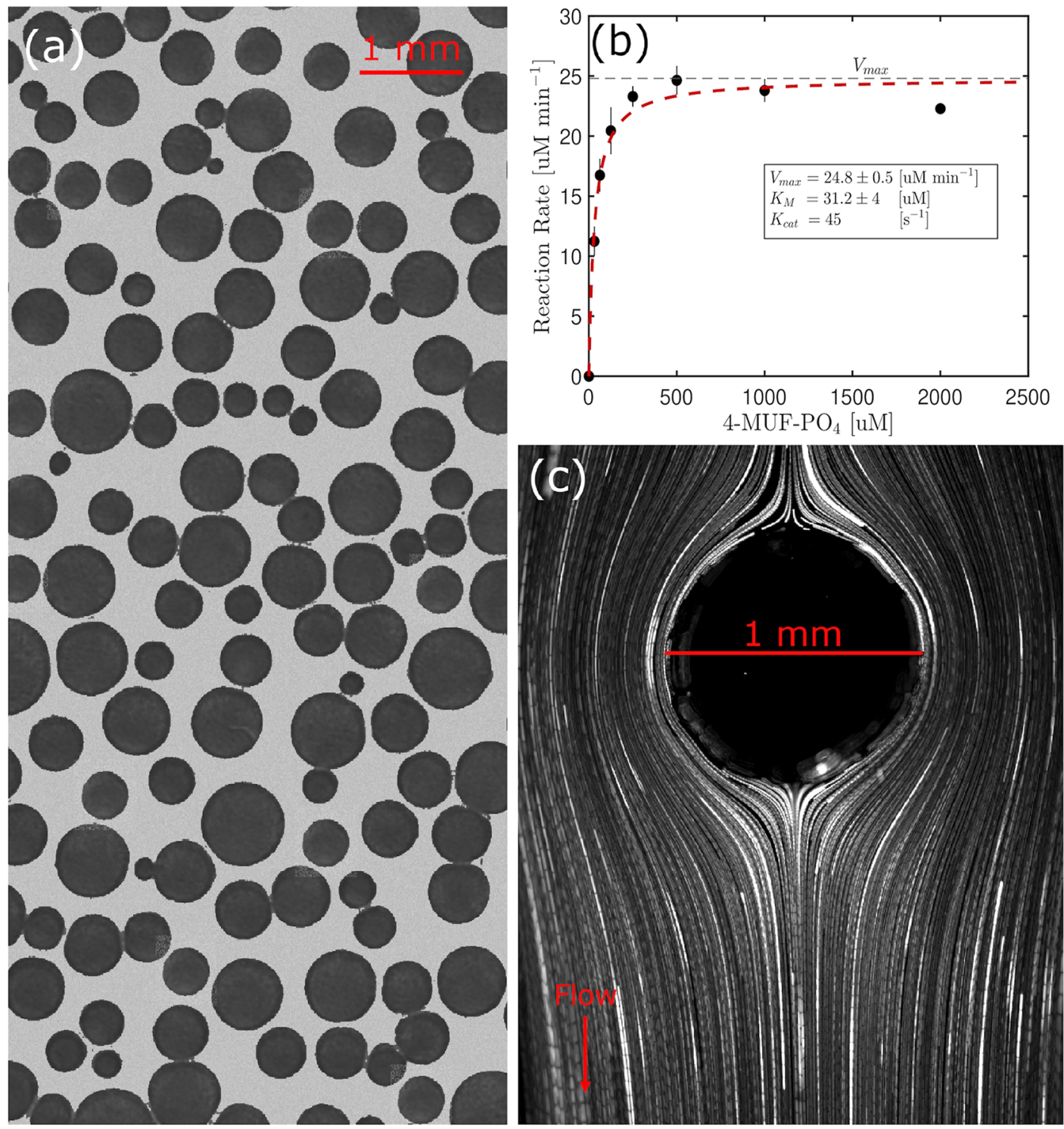

Figure 1. (a) Generated dual-porosity medium with the permeable grains (black), coated by the enzyme alkaline phosphatase, and the macropore space (gray). (b) Michaelis-Menten diagram (reaction rate) for alkaline phosphatase as a function of 4-methyl-umbelliferyl phosphate (4-MUF$\mathrm{PO}_{4}$ ) concentration (measured values, black dots; bars, standard deviation). Fitted function (red dashed line; $R^{2}=0.97$ ), where $V_{\max }$ is the maximum apparent reaction rate (black dashed line), $K_{\mathrm{M}}$ is the apparent Michaelis constant, and $K_{\text {cat }}$ (turnover number) is the maximum number of substrate molecules converted to product per enzyme molecule per second. (c) Time projection image showing trajectories of fluorescent particles ( 5 um diameter) around a single grain coated by the enzyme alkaline phosphatase $(\mathrm{Pe}=100)$.

This study focuses on reactive transport at the pore scale, in a dual-porosity domain, in which the solid phase consists of porous grains. ${ }^{5}$ Chemical reaction occurs at the interface between the invading fluid and the perimeter of the porous obstacles, without altering the solid-phase geometry. This scenario is analogous to many industrial and natural processes that take place within porous materials, such as flow and transport in biofilms in natural porous media and bioreactors, ${ }^{6-9}$ soil aggregates, ${ }^{10}$ and transport in biological tissues. $^{11,12}$ In this context, the solid phases (i.e., grains in soils and rock matrix) of natural porous media often (with some exceptions as sand grains) have subporosity (and thus secondary permeability) regions, such as silicate minerals, ${ }^{13}$ organic components, ${ }^{14}$ and carbonate rocks. ${ }^{15}$ Similarly, industrial systems such as active carbon filters used for water treatment are remarkable dual-porosity reactive systems. ${ }^{16-18}$ The principal effect of dual-porosity domains on flow and transport is to increase the residence time of the transported chemical species within the domain, ${ }^{19-21}$ which results in an amplification of anomalous transport behavior ${ }^{22,23}$ due to the contribution of lower-permeability (stagnant) zones. In these less permeable regions, diffusion dominates over advection, and thus, stagnant zones within the flow domain play a major role in the overall behavior of a reactive system.

Chemical interaction between the fluid and the solid phase in porous materials was studied extensively over the past several decades, ${ }^{24,25}$ yet most studies focused on the dynamics of precipitation/dissolution systems..$^{26-29}$ For dissolution scenarios, the spatiotemporal evolution of the system is dependent on Péclet ( $\mathrm{Pe}$, ratio between diffusive and advective time scales) and Damköhler numbers ( $\mathrm{Da}$, ratio between reaction and diffusion or advection time scales), where the mass transfer of the "active" chemical species toward the solid phase determines the evolution of the geometry of the 
system. ${ }^{30-37}$ In contrast, studies that aim to investigate the effect of various reactive-transport regimes in porous media, where chemical reactions occur at the fluid-solid interface without altering the solid phase, are rare. ${ }^{38-40}$ The few existing studies point out that the average flow rate in the system (which controls the values of $\mathrm{Pe}$ and $\mathrm{Da}$ ) affects the total amount of reaction, such that the mass production from chemical reaction decreases at high flow rates. ${ }^{41}$ Theoretical studies point out that the global fluid-solid reaction rate in porous systems is a constant, dependent on the magnitude of the fluid velocity. ${ }^{39,40}$ However, in situ observations, i.e., at the pore scale, which could improve the understanding of the role of $\mathrm{Pe}$ and $\mathrm{Da}$ in the spatial and temporal evolution of reactants and products, are still lacking.

This study explores a fluid-solid reaction (i.e., heterogeneous irreversible reaction) and the spatiotemporal evolution of the reaction product migrating through a nondeformable dual-porosity system under a wide range of $\mathrm{Pe}$ and $\mathrm{Da}$ numbers. These dimensionless numbers encompass the key physical mechanisms, i.e., advection, diffusion, and reaction, that control reactive transport in porous media. Investigation of a relatively wide range of these dimensionless numbers enables examination of the response of the system to different reactive-transport regimes. For this purpose, the interaction between solids and chemical species transported by the fluid was characterized using a unique microfluidic fabrication technique that enables enzyme (acting as one of the reactants) immobilization on the edge of the solid phase. For a given Da, it is shown that the temporal evolution of the reactivetransport system is determined by the value of Pe. Using numerical simulations calibrated against the experiments, a wider range of $\mathrm{Pe}$ and $\mathrm{Da}$ values is then explored to generalize the findings.

\section{MATERIALS AND METHODS}

2.1. Micromodel Fabrication and Experimental Protocol. A microfluidic chip (Sticky-Slide VI 0.4, IBIDI) was used, with overall dimensions of $1.7 \mathrm{~cm} \times 0.38 \mathrm{~cm} \times 0.04$ $\mathrm{cm}$ in the $x, y$, and $z$ directions, respectively. The "solid" phase was generated using a mixture of a hydrogel, consisting of $20 \%$ (w/v) acrylamide, $1 \%(\mathrm{w} / \mathrm{v})$ bis-acrylamide, $20 \mathrm{mg} / \mathrm{mL}$ photoinitiator 4-(2-hydroxyethoxy)phenyl 2-hydroxy-2-propyl ketone (Irgacure D-2959), and a protein linker ( $N$ acryloxysuccinimide, $50 \mathrm{mM}$ ). The protein linker was crudely made by reacting $1 \mathrm{mmol}$ of sodium acrylate with $1 \mathrm{mmol}$ of 1 ethyl-3-(3-dimethylaminopropyl)carbodiimide hydrochloride (EDC) and $1.1 \mathrm{mmol}$ of $\mathrm{N}$-hydroxysulfosuccinimide (NHS) in $100 \mathrm{mM}$ 4-morpholineethanesulfonic acid (MES buffer) at $\mathrm{pH}$ 6.5, for $1 \mathrm{~h}$, at room temperature. The linker is covalently bound to the acrylamide polymer within the hydrogel; it has the ability to covalently bind through primary amine groups to enzymes in the solution. To cast the solid phase, a hydrogel mixture was injected into the microfluidic chip and a mask with the negative image of the chosen geometry was placed above it. The hydrogel was then solidified by being exposed to a mercury light source for $20 \mathrm{~s}$ (Figure 1a) (Nikon LHM100C-1 instrument connected to a $100 \mathrm{~W}$ CHIU technical corporation power supply). The mask was printed with randomly sized, distributed circles (solid "grains"), with an average radius of $200 \mathrm{um}$ and a variance of $50 \mathrm{um}$. The residual unreacted hydrogel was rinsed by injecting a buffer solution [50 mM Tris- $\mathrm{HCl}(\mathrm{pH} 8.0)$ and $100 \mathrm{mM} \mathrm{NaCl}$ in doubly distilled water]. The resulting dual-porosity/dual-permeability porous medium had a macroscopic porosity $\phi$ of 0.5 , excluding the subporosity of the hydrogel grains, $\phi_{\mathrm{h}}$ (Table S1). Finally, an enzyme solution of Shrimp alkaline phosphatase $(0.3 \mathrm{mg} /$ $\mathrm{mL}$ in buffer solution, NEB M0371) was injected into the microfluidic chip. The enzyme solution was incubated within the chip for $1 \mathrm{~h}$ to covalently bind the hydrogel grains to produce a chemically reactive surface at the grain perimeter (only at the grain surface, not inside the grain).

The coated chip was then used to run the fluid-solid reactive experiments (see above). Note that to avoid potential changes in the microfluidic chip final configuration, we reused the same chip for each set of experiments (i.e., sections 3.1 and $3.2)$; after the conclusion of each experiment, the chip was flushed with a background solution until no signal could be detected by fluorescence microscopy. The microfluidic chip was initially filled with a buffer solution. The experiments started with the injection of the substrate 4-methyl-umbelliferyl phosphate (4-MUF- $\left.\mathrm{PO}_{4}\right)$. The alkaline phosphatase $\left(C_{0}=500\right.$ um), which is immobilized on the grain surfaces, catalyzes the hydrolysis of 4-MUF- $\mathrm{PO}_{4}$ to phosphate and the fluorescent compound 4-methylumbelliferone. In the following analysis, and for the sake of simplicity, the reaction is summarized as $\mathrm{A}_{\mathrm{aq}}$ $\left(4-\mathrm{MUF}-\mathrm{PO}_{4}\right)+\mathrm{B}_{\mathrm{s}}$ (alkaline phosphatase) $\rightarrow \mathrm{C}_{\mathrm{aq}}$ (4methylumbelliferone). The Michaelis-Menten model was adopted to describe the enzyme kinetics, i.e., the rate of enzymatic reaction (Figure $1 \mathrm{~b}$ ). The enzymatic rates were measured using a 96-well plate reader (BioTek Instruments). All reactions were carried out in $200 \mathrm{uL}$ of a buffer solution supplemented with $0.5 \mathrm{ug} / \mathrm{mL}$ Shrimp alkaline phosphatase (NEB M0371) and different concentrations of 4-MUF-PO The reaction was monitored using the fluorescence signal (excitation at $375 \pm 28 \mathrm{~nm}$ and emission at $460 \pm 60 \mathrm{~nm}$ ), which was then compared to the signal obtained from known concentrations of 4-MUF-PO $\mathrm{P}_{4}$. Kinetic parameters were obtained by fitting the initial velocities with a classic Michaelis-Menten model.

The reaction product was monitored by fluorescence microscopy with an excitation wavelength of $375 \pm 28 \mathrm{~nm}$ and an emission wavelength of $460 \pm 60 \mathrm{~nm}$; experiments were visualized using a Nikon (Eclipse TI-2) microscope at $4 \times$ magnification, capturing phase and fluorescence images at 100 ms intervals using an Orca flash 4.0 (Hamamatsu) camera. Images were analyzed using NIS-Elements 4.40 .00 (Nikon) and the Matlab image analysis toolbox. The experiments were concluded by injecting a background solution to measure the dynamics of the flushing process. The spatial intensity of light was converted into concentration through a calibration curve (Figure S1). ${ }^{42}$ The global measure of the chemical kinetics (i.e., global rate of reaction production) was evaluated by monitoring the accumulation of mass within the domain $(V)$ of the reaction product $(C)$ over time: ${ }^{43}$

$$
M_{\mathrm{C}}(t)=\int_{V} C_{\mathrm{C}}(t, \mathbf{x}) \mathrm{d} \mathbf{x}
$$

Note that in eq 1 , we use a uniform concentration profile along the $z$ axis. To determine the impact of fluid velocities on the spatiotemporal evolution of the reactive system, three different flow rates were imposed using a syringe pump (Harvard Apparatus Inc.). Solution A was injected for 5 characteristic time units $\tau_{\mathrm{c}}\left(\tau_{\mathrm{c}}=t \bar{v}_{x} / L_{x}\right)$, where $\bar{v}_{x}$ is the average fluid velocity in the $x$ direction (parallel to the pressure gradient), $L_{x}$ is the dimension of the domain in the same direction, and $t$ is the observation time. The flow, transport, 

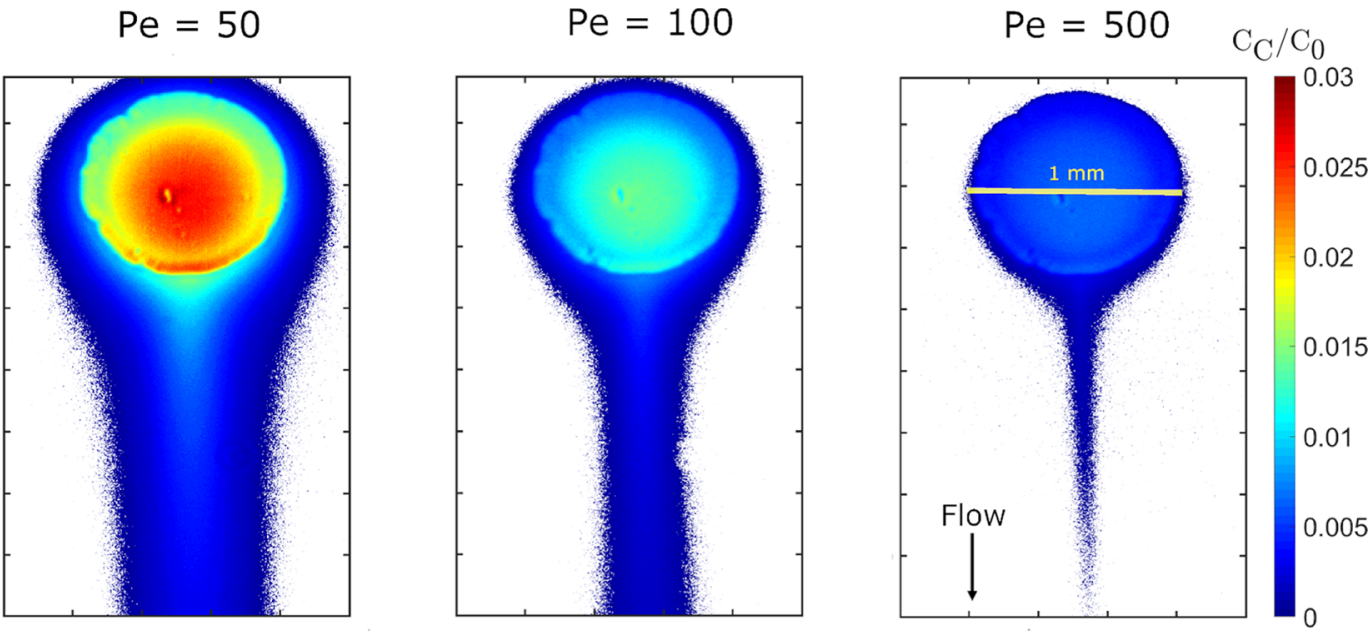

Figure 2. Normalized concentration field $\left(C_{C} / C_{0}\right)$ of reaction product $C$ at a $\tau_{\mathrm{c}}$ of 5 for each Pe value studied $(50,100$, and 500 from left to right, respectively) in the single-grain experimental system. Note that the grain diameter is $1 \mathrm{~mm}$, with a resolution of $0.0018 \mathrm{~mm}$ per pixel and a Da of 0.2. The white background indicates a concentration below the detection limit.

and reactive regimes were characterized by three dimensionless numbers: (i) Reynolds number, which characterizes the ratio between inertial and viscous forces, defined here as $\operatorname{Re}=\bar{v} l / \nu$, where $\nu$ is the kinematic viscosity of the fluid, $l$ is the average diameter of the grains (typical system length scale), and $\bar{v}$ is the average fluid velocity, (ii) Péclet number, defined as the ratio between diffusion and advection time scales (over the typical system length scale), $\mathrm{Pe}=\frac{\bar{v} l}{D}$, where $D$ is the molecular diffusion coefficient, and (iii) Damköhler number, defined as the ratio between diffusion and reaction time scales, $\mathrm{Da}=\frac{k l^{2}}{\mathrm{C}_{0} D}$, where $k$ is the enzyme reaction rate. Note that for the enzyme, the reaction rate depends on the prescribed value of the reactant species concentration $\left(C_{\mathrm{A}}\right)$ (Figure $1 \mathrm{~b}$ ); $k\left(C_{0}\right)$ was used in the characterization of Da.

2.2. Modeling Framework. To expand the range of $\mathrm{Pe}$ and $\mathrm{Da}$ values, i.e., to investigate a wider range of transport conditions beyond the limitations imposed by the experiments, e.g., acquisition frequency of the camera and motion stage displacement at high flow rates, two-dimensional numerical simulations were performed using COMSOL Multiphysics. The numerical model was first validated against the experimental results.

The Brinkman equation, ${ }^{44}$ which describes the flow through the larger pores using the Navier-Stokes equations and through the hydrogel permeable obstacles by a continuum approach (Darcy), was used to fully determine the fluid flow in the microfluidic chip. The impact of the third dimension, i.e., no-slip conditions at the upper and lower boundaries of the microfluidic chip, on the velocity field and transport was modeled by introducing a Darcy-like term to account for the drag force exerted by the upper and lower walls. ${ }^{45}$ The flow was dominated by the viscous term $(\operatorname{Re}<1)$, and thus for the macropores, the Navier-Stokes equations can be reduced to the Stokes equation $\nabla p=\mu \nabla^{2} \mathbf{v}$, where $\nabla p$ is the pressure gradient and $\mu$ is the fluid dynamic viscosity, coupled with mass conservation, $\nabla \mathbf{v}=0 .{ }^{46}$ Within the hydrogel grains, the flow field was obtained by solving Darcy's law: $\mathbf{v}_{\mathrm{h}}=-(\kappa /$ $\mu) \nabla \mathbf{I} \phi_{\mathrm{h}}$, where $\mu$ and the hydrogel permeability $(\kappa)$ control the fluid average velocity $\left(\mathbf{v}_{\mathrm{h}}\right)$ under an external driven gradient (I). This expression follows directly from the Navier-Stokes equations by assuming that the inertial effects and terms that express energy dissipation can be neglected as a result of momentum transfer within the fluid. ${ }^{46}$

Chemical transport in the system was modeled using the advection-diff usion-reaction equation, $\frac{\partial C_{\mathrm{i}}}{\partial t}+\mathbf{v} \nabla C_{\mathrm{i}}-D \nabla^{2} C_{\mathrm{i}}=r\left(C_{\mathrm{A}}, C_{\mathrm{B}}\right)$, where $C_{\mathrm{i}}$ is the chemical species concentration (i.e., $\mathrm{A}, \mathrm{B}$, or $\mathrm{C}$ ) and $r\left(C_{\mathrm{A}}, C_{\mathrm{B}}\right)$ is the rate of product creation via reaction. Note that due to the low fluid flow velocity inside the hydrogel, we neglected the mechanical dispersion mechanism and assumed that the dispersion is controlled by molecular diffusion, i.e., a diffusive regime. A unit step (Heaviside) injection condition was used to determine the temporal evolution of the reactive system. Reactant species A was introduced into the system via pulse injection from the left boundary $\left[C_{A}\left(0, t \leq 5 \tau_{c}\right)=C_{0}\right]$, analogous to the experiments. A first-order kinetic was considered at the fluid-solid interface (i.e., enzyme is only at the grain surface, not inside the grain), such that, $J_{\mathrm{r}}=k C_{\mathrm{w}}$, where $J_{\mathrm{r}}$ is the local reactive flux (normal to the solid grains), ${ }^{39} k=\frac{V_{\max }}{K_{\mathrm{M}}+C_{\mathrm{w}}}$ is the local enzyme reaction rate, $C_{\mathrm{w}}$ is the concentration of $\mathrm{A}$ on the fluid-solid interface, and $V_{\max }$ and $K_{\mathrm{M}}$ are the maximum apparent reaction rate and the apparent Michaelis constant, respectively. The first represents the maximum production rate of a system under a saturating substrate concentration, and the second is the substrate concentration at which the reaction rate is half of $V_{\max }$. To validate the numerical simulations against the experimental results, a single "free" parameter is needed. For this purpose, we used $E_{0}=V_{\text {max }} / K_{\text {cat }}$ which represents the enzyme concentration on the solid surface, and where $K_{\text {cat }}$ (turnover number) is the maximum number of substrate molecules converted to product per enzyme molecule per second. The values of Michaelis-Menten parameters are shown in Figure $1 \mathrm{~b}$. Using the numerical simulations, the control of $\mathrm{Pe}$ and $\mathrm{Da}$ over the normalized global reaction rate $\left(\bar{R}_{\mathrm{C}}=\frac{J \tau_{\mathrm{c}}}{C_{0}}\right.$, where $J$ is the total flux of the system) in the porous system was studied systematically.

\section{RESULTS AND DISCUSSION}

3.1. Fluid-Solid Reaction in a Single-Grain System. To gain a better understanding of the fluid-solid reaction 
dynamics, observation of the system at the scale of a single grain enables study of the concentration field at a very high resolution, as well as the system response to different transport conditions (Pe values). In this case, the grain has a radius of $500 \mathrm{um}$. Figure 2 shows the spatial concentration of the reaction product $\left(C_{\mathrm{C}}\right)$ at steady-state production, under three different Pe values $(50,100$, and 500). For a given $\mathrm{Da}$ (0.2 for all cases), the Pe value controls the amount of reaction and the spatial spreading of the transported C. For a reaction to occur, reactant species A must physically interact with the enzymes on the surface of the solid containing reactant species B. Reaction product $\mathrm{C}$ is then transported by diffusion from the obstacle (grain) surface into the surrounding fluid streamlines, and also through the obstacle (permeable). At the solid surface, the advective component is almost absent, as shown by particle trajectories in this system (Figure 1c). Because $\mathrm{Da}<1$, diffusion is the dominant process controlling the fluid-solid reaction. We can therefore define the reactive system as transport-dominant under the given conditions. As a result, a lower $\mathrm{Pe}$ leads to more production and a higher global concentration $C_{\mathrm{C}}$ (Figure 2), and also to wider spreading around the obstacle. The spatial distribution of product $C$ exhibits a narrower tail as Pe increases, because of the weaker contribution of molecular diffusion versus advection, i.e., diffusion between streamlines (Figure 2). This spatial difference in transport spreading must be taken into consideration when attempting to quantify reactive transport, by upscaling, in a similar physical system, such that the parameters that characterize the transport should be modified according to the value of Pe.

Figure 3 shows the normalized average concentration of product $\mathrm{C}$ (eq 1) for the single-grain experiments shown in Figure 2. Overall, a similar temporal evolution is observed for the different $\mathrm{Pe}$ values, with an increase in product concentration until a steady-state condition is reached, a plateau during the steady-state condition, followed by a decline due to the switch to the background solution at $5 \tau_{\mathrm{c}}$. Note that the effect of $\mathrm{Pe}$ is examined under normalized time $\left(\tau_{\mathrm{c}}\right)$. This representation ensures that the comparison among different $\mathrm{Pe}$ values is conducted under a fixed mass of reactant A injected into the system. As such, the specific time for each experiment is proportional to the value of $\mathrm{Pe}$ (by changing the average fluid velocity). The differences in the temporal evolution of $C_{C}$ within the system show two important features in response to an increase in Pe: (i) the product concentration $C_{C}$ under steady-state conditions within the domain is reduced, and (ii) the system exhibits longer tailing of $\mathrm{C}$ mass recovery during the flushing with the background solution. Both features arise from the role of molecular diffusion, as it controls the ability of reactant species $A$ to interact with the solid phase coated with $\mathrm{B}$, and the ability of reaction product $\mathrm{C}$ to diffuse away from, and to pass through and leave, the permeable grains; thus, both phenomena are determined by the value of Pe.

The numerical model was validated against these experimental results. The results from the numerical simulations are depicted as solid lines in Figure 3. Note that the parameters used in the numerical simulations were taken from the experimental data, and the only free parameter is the enzyme concentration on the solid surface $\left(E_{0}\right)$. Details of parameters used to validate the model can be found in Table S1.

3.2. Fluid-Solid Reactive Transport in Porous Media. For porous media, in contrast to single-grain systems, the transport product (following reaction) interacts with multiple

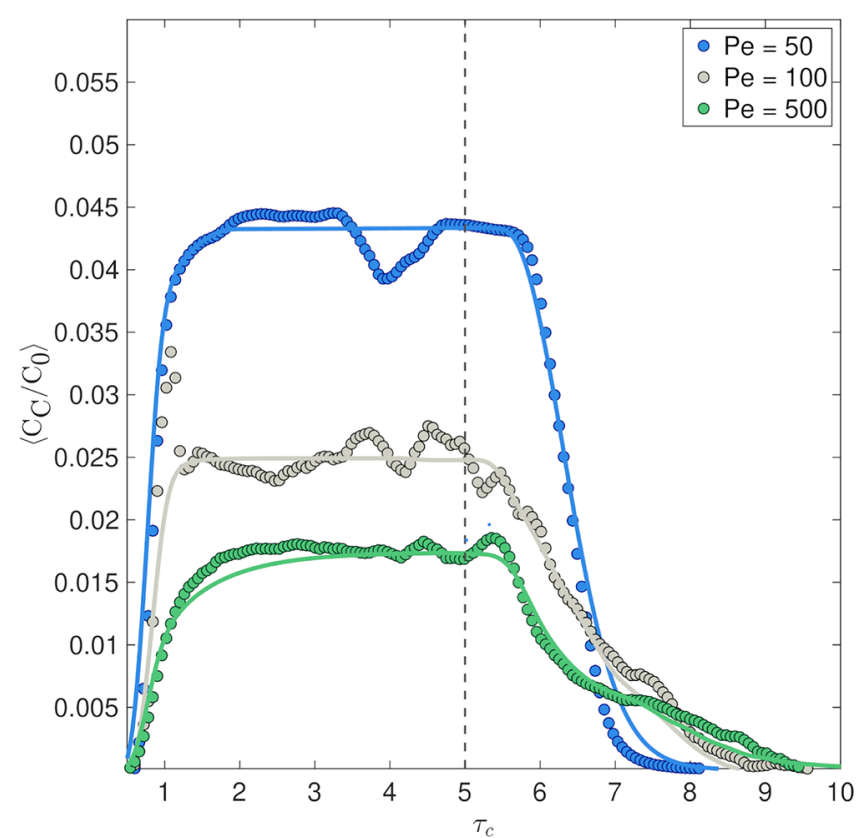

Figure 3. Temporal evolution of the normalized average concentration of the reaction product $\left(\left\langle C_{\mathrm{C}} / C_{0}\right\rangle\right)$ at a given Da value $(0.2)$ for the Pe values $(50,100$, and 500) studied in the single-grain system experiments (dots). The vertical dashed line represents the switch at the inlet from $\mathrm{A}$ to the background solution for the flushing stage. Solid lines (same color criteria as for experiments) show the numerical modeling results calibrated against the experimental results. Note that the image resolution is of $0.0018 \mathrm{~mm}$ per pixel.

permeable obstacles, which increases the complexity of the transport behavior. Figure 4a shows the temporal evolution of the normalized average concentration of $\mathrm{C}$; as seen for the single-grain results, the pattern of temporal evolution is determined by the value of Pe. However, in this case, the time to reach steady-state production of $\mathrm{C}$ differs among $\mathrm{Pe}$ values. This highlights the importance of the diffusive motion of $\mathrm{C}$ within the permeable obstacles. Panels $\mathrm{b}$ and $\mathrm{c}$ of Figure 4 show the experimental observations of the concentration field of $C_{C}$ at a $\tau_{c}$ of 5 , having higher concentrations $C_{C}$ as $\mathrm{Pe}$ decreases. Different flow regions can be distinguished from the concentration fields: (i) well-connected flow paths, wherein the advective component is dominant, and (ii) low-velocity regions, in which the diffusive component dominates the transport. In addition, as the transported product continues to interact with the hydrogel pillars, its concentration downstream increases with the flow direction (Figure $4 b, c$ ).

To assess the way in which the permeable obstacles control the production and transport of the reaction product $C$, numerical simulations were carried out to compare a dualporosity system and a single-porosity system; for the latter, the obstacles (grains) are impermeable. Slip and no-slip conditions for flow at the obstacle surfaces were considered, respectively. Figure 5a shows the temporal evolution of the normalized average concentration within the two systems. The insets show the concentration field at a $\tau_{\mathrm{c}}$ of 5 for both systems. A similar temporal evolution is observed until steady-state mass production is reached. However, for $\tau_{c}$ values of $>5$, when the background solution is injected to flush the systems, the concentration tails show different behavior due to the contribution of the permeable obstacles as stagnant zones, i.e., increasing the residence time of $\mathrm{C}$ within the domain. The 

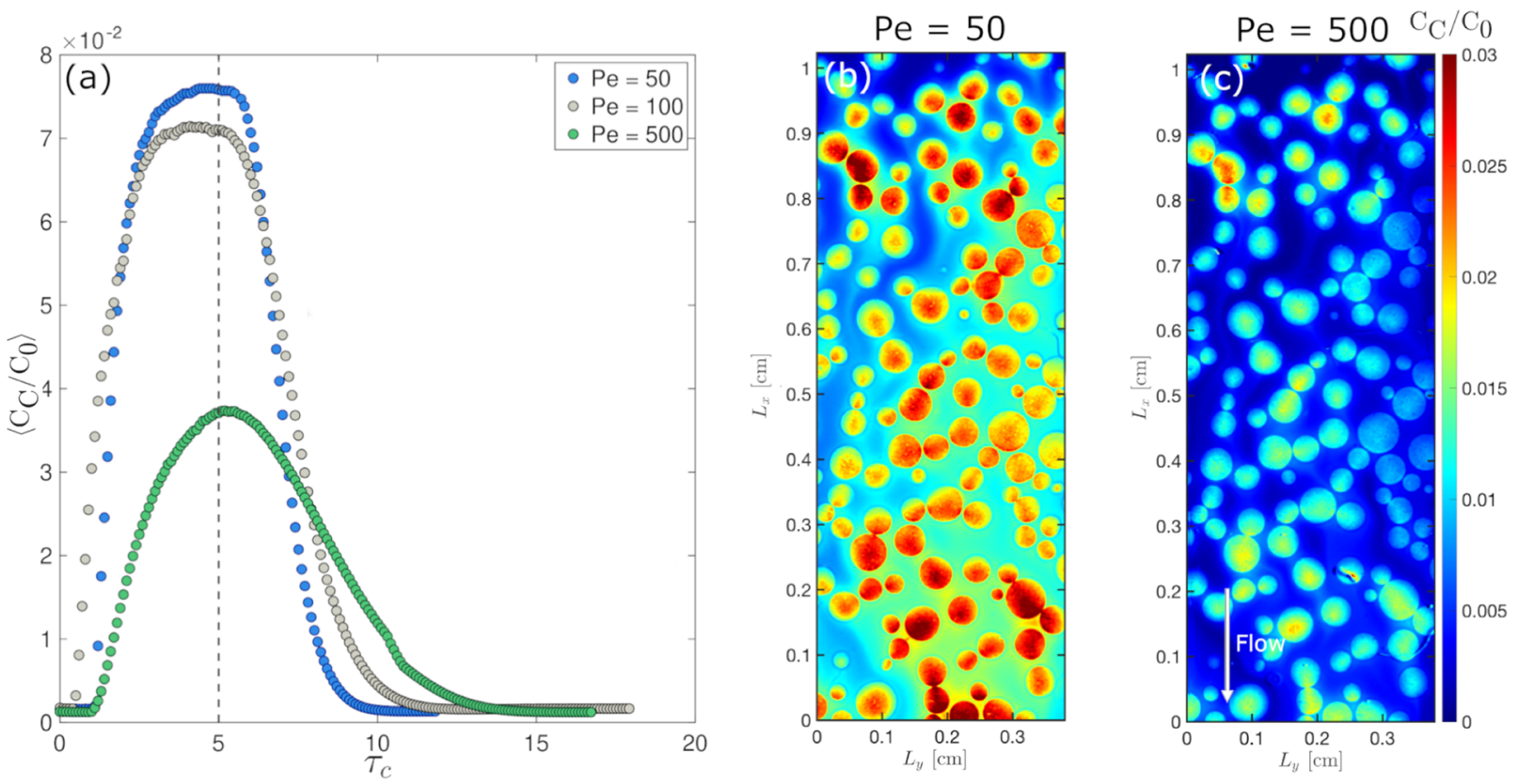

Figure 4. (a) Temporal evolution of the normalized average concentration of the reaction product $\left(\left\langle C_{\mathrm{C}} / C_{0}\right\rangle\right)$ at a given Da value $(0.2)$ for the Pe values $(50,100$, and 500) studied in the porous medium system experiments (dots). The vertical dashed line represents the switch at the inlet from A to the background solution for the flushing stage. ( $\mathrm{b}$ and $\mathrm{c}$ ) Normalized concentration field $\left(\mathrm{C}_{\mathrm{C}} / C_{0}\right)$ of reaction product $\mathrm{C}$ at a $\tau_{\mathrm{c}}$ of 5 for Pe values of 50 and 500, respectively. Note that the flow direction is downward.
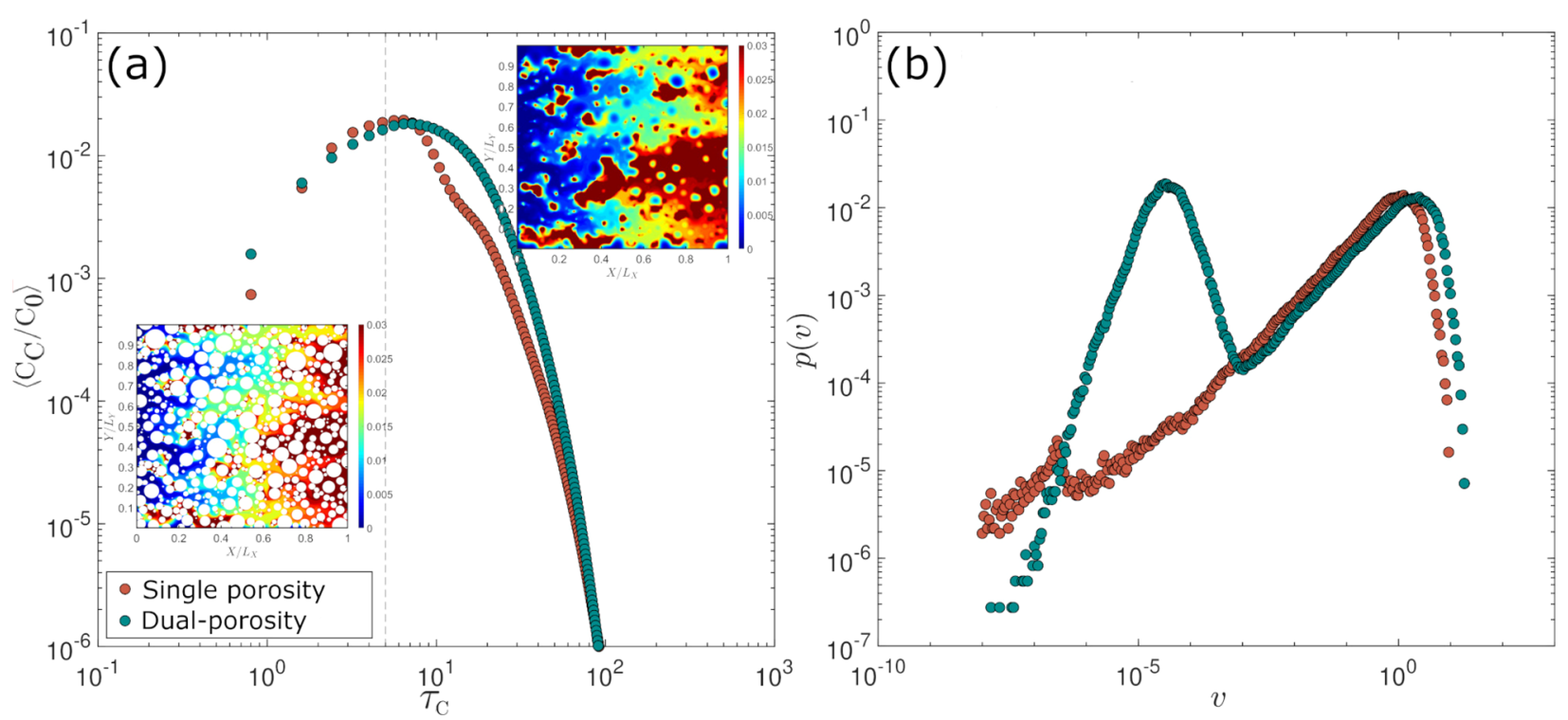

Figure 5. (a) Numerical simulations showing the evolution of the normalized average concentration within the domain of the reaction product $\left(\left\langle C_{\mathrm{C}} / C_{0}\right\rangle\right)$ at a given $\mathrm{Da}(0.2)$ and $\mathrm{Pe}(50)$, for a system with impermeable grains (single porosity, bottom left inset) and for a dual-porosity (permeable grains) system (top right inset). The insets show the normalized concentration field $\left(C_{\mathrm{C}} / C_{0}\right)$ of reaction product $\mathrm{C}$ at a $\tau_{\mathrm{c}}$ of 5 ; the flow direction is from left to right. (b) Velocity magnitude probability density function $p(v)$ in the single- and dual-porosity systems.

differences between the two systems can be understood by examining the probability density function $p(v)$ of simulated Eulerian velocity magnitudes $(v)$ (Figure $5 \mathrm{~b}$ ). For the dualporosity system, $p(v)$ becomes broader; i.e., the probability of both high and low velocities increases with the presence of permeable obstacles. The distributions show a peak at high velocities before falling off exponentially, with a slight shift toward higher velocities (i.e., increase in the characteristic velocity) with the inclusion of permeable obstacles. The dualporosity system shows an additional peak at lower velocities, corresponding to the permeable obstacles, which causes the overall increase in the residence time of the transported $C_{C}$.

3.3. Control of the Fluid-Solid Reaction Rate by $\mathrm{Pe}$ and Da. Numerical simulations are used to examine the system response to different reactive-transport regimes (i.e., beyond the limitations of the transport-dominated regime of the experimental setup) and the control of $\mathrm{Pe}$ and $\mathrm{Da}$ over the normalized global reaction rate, $\bar{R}_{\mathrm{C}}$ (i.e., the production of $\mathrm{C}$ per unit of characteristic time $\tau_{c}$ ). In most environmental and industrial applications, advection is the dominant transport process and the fluid-solid reaction is kinetically limited. 
Therefore, the following ranges were chosen for the dimensionless numbers: $1<\mathrm{Pe}<10^{4}$ and $10^{-4}<\mathrm{Da}<1$. Note that from the definition of $J_{\mathrm{r}}$, when production and concentration fields reach a steady-state spatial distribution, the concentration $C_{\mathrm{A}}$ at the grain walls approaches $C_{0}$, so that $\bar{R}_{\mathrm{C}} \rightarrow \frac{V_{\max } \tau_{\mathrm{c}}}{K_{\mathrm{m}}+C_{0}}$. The steady-state condition represents the maximum potential (efficiency) for fluid-solid reaction within the system, which is highly relevant for many environmental and industrial applications such as bioremediation and water treatment. Figure 6 displays $\bar{R}_{\mathrm{C}}$ as a function of $\mathrm{Pe}$, for

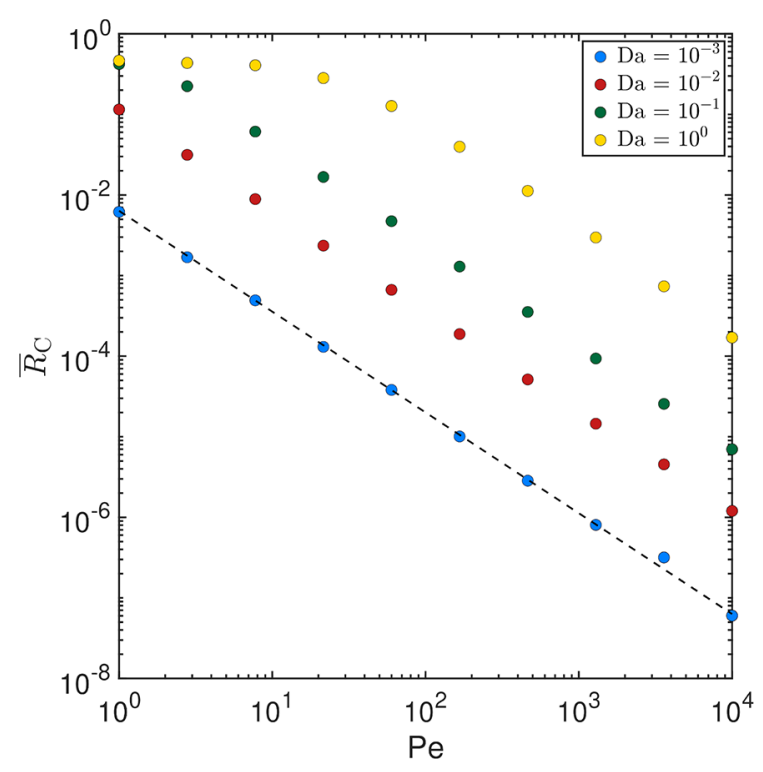

Figure 6. Normalized reaction rate $\left(\bar{R}_{\mathrm{C}}\right)$ within the porous medium system at the steady-state production rate of $\mathrm{C}$ as a function of $\mathrm{Pe}$ and Da values. Results obtained numerically.

different values of $\mathrm{Da}$ (colors). Because $\tau_{\mathrm{c}}$ and $\mathrm{Pe}$ are proportional to each other (by the fluid average velocity), a linear scaling $\bar{R}_{\mathrm{C}} \sim \mathrm{Pe}^{-1}$ is observed. However, this relation is not valid for high $\mathrm{Da}$ values and low Pe values. For these regimes, $\bar{R}_{\mathrm{C}}$ is below the mass transfer limitation (i.e., the enzyme efficiency is limited by the supplied substrate), which reduces the actual reaction rate compared to that expected from the scaling behavior. At low $\mathrm{Da}$ values, where the enzymes are operating at the maximum rate, and the reaction is not limited by mass transfer, the linear relation is wellestablished throughout the entire Pe range. In the latter, the differences between $\mathrm{Pe}$ values are due to the actual residence time of reaction product $\mathrm{C}$ within the domain, and the fact that the local reactive flux $\left(J_{\mathrm{r}}\right)$, in the steady state, is constant with respect to Pe.

However, natural systems, in which boundary conditions often change over time, ${ }^{47}$ rarely exhibit steady-state flow and transport conditions. Therefore, in addition to the steady-state analysis presented above (Figure 6), the time-dependent regimes (i.e., the time to reach a steady-state condition and the time to flush the reaction product from the system) are also studied. As shown in the experimental results (Figure 4), the time to reach steady-state mass production of $\mathrm{C}$ depends on the $\mathrm{Pe}$ value. Numerical simulations demonstrate how the combination of different $\mathrm{Pe}$ and $\mathrm{Da}$ values affects the normalized time $\left(\tau_{\mathrm{c}}^{*}\right)$ required to reach the steady state of mass production (Figure 7a). The characteristic time required to reach steady state increases as Pe increases, as previously observed in fluid-fluid systems. ${ }^{42,43}$ Two factors control this behavior: (i) the time needed for reactant A to reach a steadystate spatial distribution and (ii) the diffusive motion of product $\mathrm{C}$ within the permeable obstacles. Two regions (i.e., different slopes) can be distinguished. For advective-dominant transport $(\mathrm{Pe}>10), \tau_{\mathrm{c}}^{*}$ scales as $\tau_{\mathrm{c}}^{*}=1.5 \mathrm{Pe}^{0.55}$. For low $\mathrm{Pe}$ values $(<10)$, there is a transition zone from a strongly diffusively controlled system to the advective regime. ${ }^{48}$ For a given Pe, the magnitude of the impact of the Da value on $\tau_{\mathrm{c}}^{*}$ is much smaller (Figure $7 \mathrm{a}$ ), where $\tau_{\mathrm{c}}^{*}$ slightly increases as Da
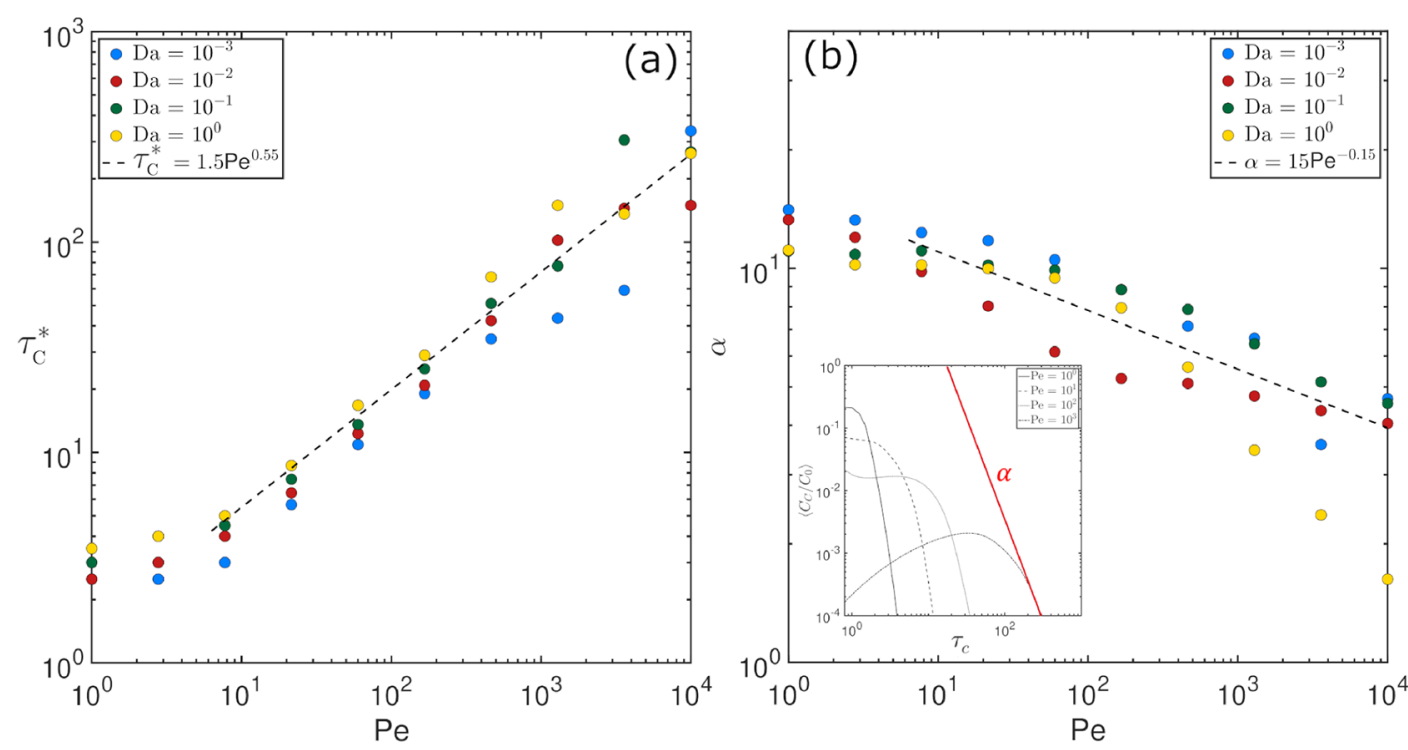

Figure 7. (a) Number of $\tau_{\mathrm{c}}^{*}$ to reach a steady-state condition of $\mathrm{C}$ production in the porous medium system. (b) Rate $\alpha$ at which reaction product $\mathrm{C}$ is flushed from the system. The inset shows the temporal evolution of the normalized average concentration of the reaction product $\left(\left\langle C_{\mathrm{C}} / C_{0}\right\rangle\right)$ and the impact of $\mathrm{Pe}$ on the $\alpha$ value $\left(\mathrm{Da}=10^{-1}\right)$. Results obtained numerically. 
increases, although introducing a larger variability for Pe values of $>10^{3}$.

An additional important feature that arises from the experimental results is the temporal evolution of concentration when reaction product $C$ is flushed from the system (Figure 4). During the flushing stage, the tailing of the concentration drop can be scaled by a power law with an exponent $\alpha{ }^{49}$ To obtain $\alpha$, we use a power-law function to fit the tailing shape of the concentration drop (see the red line in the inset of Figure $7 \mathrm{~b}$ as an example). The effect of $\mathrm{Pe}$ and $\mathrm{Da}$ on the fitted value of $\alpha$ (Figure 7b) is as follows: $\alpha$ decreases as Pe increases, such that the rate of flushing from the system decreases with Pe. The relation between $\mathrm{Pe}$ and $\alpha$ follows a power-law function ( $\alpha=$ $\left.15 \mathrm{Pe}^{-0.15}\right)$. Note that this scaling is an average behavior and ignores the role of $\mathrm{Da}$ (using the mean value of each $\mathrm{Da}$ ), being valid for advective-dominant regimes $(\mathrm{Pe}>10)$. No clear impact of $\mathrm{Da}$ on $\alpha$ is recognized. While for Pe values of $<10$, similar to the case for $\tau_{\mathrm{c}}^{*}$ (Figure 7a), $\alpha$ increases as $\mathrm{Da}$ decreases, for Pe values of $>10$, this pattern is not respected, scattering $\alpha$ values for a give Pe.

The results of this study demonstrate the importance of the reactive-transport regime-in particular, the specific combination between $\mathrm{Pe}$ and $\mathrm{Da}$ - on the spatiotemporal evolution of the reaction product in fluid-solid reactive systems. This study highlights the significance of stagnant zones within the flow field, in this particular case because of a dual-porosity system. These diffusive control regions affect the spatial distribution of production and reaction product (Figure 4), and their temporal evolution (Figures 3 and 4), by increasing the solute residence time within the flow domain. Similar to the effect of increasing the medium heterogeneity, the occurrence of dual porosity leads to a complex flow pattern. The proposed nonlinear scaling laws (Figures 6 and 7) give new insights into the response of such systems to fluid-solid reactive transport and can be used to assess the key parameters that control the spatiotemporal evolution of the system. The obtained results are relevant for various applications, such as the use of activated carbon in drinking water and wastewater, ${ }^{18}$ the use of pellets in industrial applications, ${ }^{50}$ and the assessment of reactive transport in natural soils and aquifers. ${ }^{36}$ As demonstrated in this work, chemical reactions on surfaces and the transport behavior of the reaction products vary as a function of Pe and Da, exhibiting different spatial and temporal patterns that do not scale linearly. Therefore, to quantify and assess both the reactivity and the transport of reaction products in heterogeneous porous media, this nonlinear variation of the physical parameters controlling them should be taken into consideration.

\section{ASSOCIATED CONTENT}

\section{s) Supporting Information}

The Supporting Information is available free of charge at https://pubs.acs.org/doi/10.1021/acsestwater.0c00043.

Calibration curves (intensity vs concentration) and validation of the numerical simulations from experimental observations (PDF)

\section{AUTHOR INFORMATION}

\section{Corresponding Authors}

Uria Alcolombri - Department of Civil, Environmental and Geomatic Engineering, ETH Zurich, 8092 Zürich, Switzerland; Email: alcolombri@ifu.baug.ethz.ch
Joaquin Jimenez-Martinez - Department of Civil, Environmental and Geomatic Engineering, ETH Zurich, 8092 Zürich, Switzerland; Department of Water Resources and Drinking Water, Swiss Federal Institute of Aquatic Science and Technology, 8600 Dübendorf, Switzerland; (1) orcid.org/0000-0002-2063-6490;

Email: joaquin.jimenez@eawag.ch, jjimenez@ethz.ch

\section{Authors}

Alon Nissan - Department of Earth and Planetary Sciences, Weizmann Institute of Science, Rehovot 7610001, Israel; Department of Civil, Environmental and Geomatic Engineering, ETH Zurich, 8092 Zürich, Switzerland; Department of Water Resources and Drinking Water, Swiss Federal Institute of Aquatic Science and Technology, 8600 Dübendorf, Switzerland; 이이.org/0000-0003-13005591

Frédéric de Schaetzen - Department of Civil, Environmental and Geomatic Engineering, ETH Zurich, 8092 Zürich, Switzerland

Brian Berkowitz - Department of Earth and Planetary Sciences, Weizmann Institute of Science, Rehovot 7610001, Israel; (1) orcid.org/0000-0003-3078-1859

Complete contact information is available at: https://pubs.acs.org/10.1021/acsestwater.0c00043

\section{Notes}

The authors declare no competing financial interest.

\section{ACKNOWLEDGMENTS}

A.N. and J.J.-M. acknowledge the financial support from the Department of Water Resources and Drinking Water at Eawag. A.N. and J.J.-M. also kindly acknowledge Prof. R. Stocker and E. Burmeister from the Department of Civil, Environmental and Geomatic Engineering at ETH Zurich for providing access to laboratory equipment and material. U.A. gratefully acknowledge funding from the Human Frontier Science Program (HFSP fellowship, LT001209/2017). B.B. gratefully acknowledges support by the Israel Science Foundation (Grant 485/ 16). B.B. holds the Sam Zuckerberg Professorial Chair in Hydrology. J.J.-M. gratefully acknowledges the financial support from the Swiss National Science Foundation (SNF, Grant 200021 178986) and Eawag Discretionary Funding. The authors thank the two anonymous reviewers whose valuable comments and suggestions helped to improve the manuscript.

\section{REFERENCES}

(1) Mauri, R. Dispersion, convection, and reaction in porous media. Phys. Fluids A 1991, 3, 743-756.

(2) Flemming, H. C.; Wuertz, S. Bacteria and archaea on Earth and their abundance in biofilms. Nat. Rev. Microbiol. 2019, 17, 247-260.

(3) Borer, B.; Tecon, R.; Or, D. Spatial organization of bacterial populations in response to oxygen and carbon counter-gradients in pore networks. Nat. Commun. 2018, 9, 769.

(4) Middelburg, J. J. Springer Briefs in Earth System Sciences; Springer, 2019; p 126.

(5) Bécot, F. X.; Jaouen, L.; Gourdon, E. Applications of the dual porosity theory to irregularly shaped porous materials. Acta Acust. Acust. 2008, 94, 715-724.

(6) Tanyolaç, A.; Beyenal, H. Effectiveness factor for a hollow-fiber biofilm reactor at maximum substrate consumption. Chem. Eng. J. Biochem. Eng. J. 1996, 62, 149-154.

(7) De Beer, D.; Stoodley, P.; Lewandowski, Z. Liquid flow and mass transport in heterogeneous biofilms. Water Res. 1996, 30, 2761-2765. 
(8) Stoodley, P.; Dodds, I.; De Beer, D.; Scott, H. L.; Boyle, J. D. Flowing biofilms as a transport mechanism for biomass through porous media under laminar and turbulent conditions in a laboratory reactor system. Biofouling 2005, 21, 161-168.

(9) Deng, W.; Cardenas, M. B.; Kirk, M. F.; Altman, S. J.; Bennett, P. C. Effect of permeable biofilm on micro-and macro-scale flow and transport in bioclogged pores. Environ. Sci. Technol. 2013, 47, 1109211098 .

(10) Brusseau, M. L.; Gerstl, Z.; Augustijn, D.; Rao, P. S. Simulating solute transport in an aggregated soil with the dual-porosity model: measured and optimized parameter values. J. Hydrol. 1994, 163, 187193.

(11) Wood, B. D.; Quintard, M.; Whitaker, S. Calculation of effective diffusivities for biofilms and tissues. Biotechnol. Bioeng. 2002, 77, 495-516.

(12) Truskey, G. A.; Yuan, F.; Katz, D. F. Transport phenomena in biological systems; Pearson, 2004.

(13) Brantley, S. L.; Mellott, N. P. Surface area and porosity of primary silicate minerals. Am. Mineral. 2000, 85, 1767-1783.

(14) Marinari, S.; Masciandaro, G.; Ceccanti, B.; Grego, S. Influence of organic and mineral fertilisers on soil biological and physical properties. Bioresour. Technol. 2000, 72, 9-17.

(15) Ehrenberg, S. N.; Nadeau, P. H. Sandstone vs. carbonate petroleum reservoirs: A global perspective on porosity-depth and porosity-permeability relationships. AAPG Bull. 2005, 89, 435-445.

(16) Rivas, F. J.; Beltrán, F.; Gimeno, O.; Acedo, B.; Carvalho, F. Stabilized leachates: Ozone-activated carbon treatment and kinetics. Water Res. 2003, 37, 4823-4834.

(17) Gerrity, D.; Gamage, S.; Holady, J. C.; Mawhinney, D. B.; Quiñones, O.; Trenholm, R. A.; Snyder, S. A. Pilot-scale evaluation of ozone and biological activated carbon for trace organic contaminant mitigation and disinfection. Water Res. 2011, 45, 2155-2165.

(18) Marron, E. L.; Mitch, W. A.; Gunten, U. v.; Sedlak, D. L. A tale of two treatments: The multiple barrier approach to removing chemical contaminants during potable water reuse. Acc. Chem. Res. 2019, 52, 615-622.

(19) Gerke, H. H.; van Genuchten, M. T. A dual-porosity model for simulating the preferential movement of water and solutes in structured porous media. Water Resour. Res. 1993, 29, 305-319.

(20) Andrade, J. S.; Almeida, M. P.; Mendes Filho, J.; Havlin, S.; Suki, B.; Stanley, H. E. Fluid flow through porous media: The role of stagnant zones. Phys. Rev. Lett. 1997, 79, 3901-3904.

(21) Kandhai, D.; Hlushkou, D.; Hoekstra, A. G.; Sloot, P. M. A.; Van As, H.; Tallarek, U. Influence of stagnant zones on transient and asymptotic dispersion in macroscopically homogeneous porous media. Phys. Rev. Lett. 2002, 88, 234501.

(22) Berkowitz, B.; Cortis, A.; Dentz, M.; Scher, H. Modeling nonFickian transport in geological formations as a continuous time random walk. Rev. Geophys. 2006, 44, 1-49.

(23) Bijeljic, B.; Rubin, S.; Scher, H.; Berkowitz, B. Non-Fickian transport in porous media with bimodal structural heterogeneity. $J$. Contam. Hydrol. 2011, 120-121, 213-221.

(24) Berkowitz, B.; Dror, I.; Hansen, S. K.; Scher, H. Measurements and models of reactive transport in geological media. Rev. Geophys. 2016, 54, 930-986.

(25) Seigneur, N.; Mayer, K. U.; Steefel, C. I. Reactive transport in evolving porous media. Rev. Mineral. Geochem. 2019, 85, 197-238.

(26) Singurindy, O.; Berkowitz, B.; Lowell, R. P. Carbonate dissolution and precipitation in coastal environments: Laboratory analysis and theoretical consideration. Water Resour. Res. 2004, 40, 113.

(27) Prasianakis, N. I.; Curti, E.; Kosakowski, G.; Poonoosamy, J.; Churakov, S. V. Deciphering pore-level precipitation mechanisms. Sci. Rep. 2017, 7, 13765.

(28) Hommel, J.; Coltman, E.; Class, H. Porosity-permeability relations for evolving pore space: a review with a focus on (bio-) geochemically altered porous media. Transp. Porous Media 2018, 124, 589-629.
(29) Jiménez-Martínez, J.; Hyman, J. D.; Chen, Y.; Carey, J. W.; Porter, M. L.; Kang, Q.; Guthrie, G., Jr; Viswanathan, H. S. Homogenization of dissolution and enhanced precipitation induced by bubbles in multiphase flow systems. Geophys. Res. Lett. 2020, 47, e2020GL087163.

(30) Daccord, G. Chemical dissolution of a porous medium by a reactive fluid. Phys. Rev. Lett. 1987, 58, 479-482.

(31) Daccord, G.; Lenormand, R.; Liétard, O. Chemical dissolution of a porous medium by a reactive fluid-I. Model for the "wormholing" phenomenon. Chem. Eng. Sci. 1993, 48, 169-178.

(32) Detwiler, R. L.; Glass, R. J.; Bourcier, W. L. Experimental observations of fracture dissolution: The role of Peclet number on evolving aperture variability. Geophys. Res. Lett. 2003, 30 (12), 1648.

(33) Kang, Q.; Chen, L.; Valocchi, A. J.; Viswanathan, H. S. Porescale study of dissolution-induced changes in permeability and porosity of porous media. J. Hydrol. 2014, 517, 1049-1055.

(34) Singh, R.; Yoon, H.; Sanford, R. A.; Katz, L.; Fouke, B. W.; Werth, C. J. Metabolism-induced $\mathrm{CaCO} 3$ biomineralization during reactive transport in a micromodel: Implications for porosity alteration. Environ. Sci. Technol. 2015, 49, 12094-12104.

(35) Menke, H. P.; Bijeljic, B.; Andrew, M. G.; Blunt, M. J. Dynamic three-dimensional pore-scale imaging of reaction in a carbonate at reservoir Conditions. Environ. Sci. Technol. 2015, 49, 4407-4414.

(36) Al-Khulaifi, Y.; Lin, Q.; Blunt, M. J.; Bijeljic, B. Reaction rates in chemically heterogeneous rock: Coupled impact of structure and flow properties studied by X-ray microtomography. Environ. Sci. Technol. 2017, 51, 4108-4116.

(37) Yoon, H.; Chojnicki, K. N.; Martinez, M. J. Pore-scale analysis of calcium carbonate precipitation and dissolution kinetics in a microfluidic device. Environ. Sci. Technol. 2019, 53, 14233-14242.

(38) Langner, H. W.; Inskeep, W. P.; Gaber, H. M.; Jones, W. L.; Das, B. S.; Wraith, J. M. Pore water velocity and residence time effects on the degradation 2,4-D during transport. Environ. Sci. Technol. 1998, $32,1308-1315$.

(39) Wood, B. D.; Radakovich, K.; Golfier, F. Effective reaction at a fluid-solid interface: Applications to biotransformation in porous media. Adv. Water Resour. 2007, 30, 1630-1647.

(40) Heße, F.; Radu, F. A.; Thullner, M.; Attinger, S. Upscaling of the advection-diffusion-reaction equation with Monod reaction. Adv. Water Resour. 2009, 32, 1336-1351.

(41) Marrazzo, W. N.; Merson, R. L.; McCoy, B. J. Enzyme immobilized in a packed-bed reactor: Kinetic parameters and mass transfer effects. Biotechnol. Bioeng. 1975, 17, 1515-1528.

(42) de Anna, P.; Jimenez-Martinez, J.; Tabuteau, H.; Turuban, R.; Le Borgne, T.; Derrien, M.; Méheust, Y. Mixing and reaction kinetics in porous media: An experimental pore scale quantification. Environ. Sci. Technol. 2014, 48, 508-516.

(43) De Anna, P.; Dentz, M.; Tartakovsky, A.; Le Borgne, T. The filamentary structure of mixing fronts and its control on reaction kinetics in porous media flows. Geophys. Res. Lett. 2014, 41, 45864593.

(44) Durlofsky, L.; Brady, J. F. Analysis of the Brinkman equation as a model for flow in porous media. Phys. Fluids 1987, 30, 3329-3341.

(45) Ferrari, A.; Jimenez-Martinez, J.; Borgne, T. L.; Méheust, Y.; Lunati, I. Challenges in modeling unstable two-phase flow experiments in porous micromodels. Water Resour. Res. 2015, 51, 13811400.

(46) Nissan, A.; Berkowitz, B. Inertial effects on flow and transport in heterogeneous porous media. Phys. Rev. Lett. 2018, 120, 054504.

(47) Nissan, A.; Dror, I.; Berkowitz, B. Time-dependent velocityfield controls on anomalous chemical transport in porous media. Water Resour. Res. 2017, 53, 3760-3769.

(48) Bijeljic, B.; Mostaghimi, P.; Blunt, M. J. Signature of nonFickian solute transport in complex heterogeneous porous media. Phys. Rev. Lett. 2011, 107, 20-23.

(49) Dentz, M.; Icardi, M.; Hidalgo, J. J. Mechanisms of dispersion in a porous medium. J. Fluid Mech. 2018, 841, 851-882.

(50) Sahinkaya, E.; Sahin, A.; Yurtsever, A.; Kitis, M. Concentrate minimization and water recovery enhancement using pellet 
precipitator in a reverse osmosis process treating textile wastewater. J.

Environ. Manage. 2018, 222, 420-427. 